\title{
A Model for Non-thermal Optical Emission from Radio Pulsars
}

\author{
S. A. Petrova \\ Institute of Radio Astronomy, National Academy of Sciences of \\ Ukraine, 4, Chervonopraporna St., 61002 Kharkiv, Ukraine
}

\begin{abstract}
Interpretation of the non-thermal optical emission of pulsars as a spontaneous synchrotron re-emission of the secondary particles involved in the process of resonant absorption of pulsar radio emission is suggested. Our model can explain the non-thermal high-energy luminosities and spectra of both young and middle-aged pulsars. Moreover, it implies a physical link between the radio and higher-energy emission of pulsars.
\end{abstract}

\section{Introduction}

The high-energy emission of radio pulsars (except perhaps hard gamma-rays) is commonly associated with incoherent synchrotron radiation of the relativistic plasma particles in the magnetosphere. However, up to now the problem has remained as to the physically grounded distribution function of radiating particles which would reproduce the observed properties of the high-energy emission. We suggest that the momentum evolution of the secondary plasma particles is mainly determined by resonant absorption of the incident radio photons (Lyubarskii \& Petrova 1998; Petrova 2002). Then the non-thermal high-energy emission arises naturally as a result of spontaneous synchrotron re-emission of absorbing particles (Petrova 2003). Within the framework of our model, the nonthermal emission of pulsars is physically connected with the radio emission. This is expected to have a number of observational manifestations (Petrova 2004).

\section{Evolution of Particle Momenta}

The resonant absorption of radio waves occurs in the outer magnetosphere, at distances $\sim(0.1-1) r_{L}$. In short-period pulsars - the only ones which can be considered as potential sources of non-thermal high-energy emission - for most of the radio frequency range the absorption depth is of order unity. Hence, the observed radio luminosities are not suppressed dramatically, though the energy absorbed can be large. Note that in the resonance region the cross-section of the radio beam is only a small fraction of the open field line tube and therefore the energy of the radio beam can exceed the energy of the plasma in the lightened area. Thus, the momenta of absorbing particles can increase markedly.

According to the conservation laws, the first absorbed radio photons act mainly to increase the particle pitch-angles, until the latter approach the angle of incidence of the photons, $\theta \sim 0.1$; further on, the pitch-angles are almost 
constant, while the photons absorbed increase the total particle momenta. The numerical estimates show that inside the resonance region of PSR B0656+14 the total particle momentum varies within an order of magnitude, whereas in the Crab pulsar the momentum can increase by a factor of $\sim 10^{3}$. The difference in the spatial evolution of the particle momenta is supposed to underlie the distinction between the energetic characteristics of the non-thermal high-energy emission of middle-aged and young pulsars.

\section{Synchrotron Re-Emission of the Absorbing Particles}

Let us turn to the characteristics of synchrotron radiation of the system of particles involved in the process of resonant absorption. Momentum evolution of the particles should be followed by a marked spatial dependence of the critical frequency of synchrotron radiation, $\nu_{c}=\frac{3}{4 \pi} \omega_{H} \gamma^{2} \psi$, where $\gamma$ is the particle Lorentz factor and $\psi$ the pitch-angle. Correspondingly, the maximum in a common single-particle synchrotron spectrum (at constant $\nu_{c}$ ) is substantially smeared and the resultant spectra are flat across several orders of magnitude in frequency.

The optical emission of middle-aged pulsars appears to be generated by particles with large enough momenta, $p \gg p_{0}$, and considerable pitch-angles, $\psi \sim \theta$, in the upper part of the resonance region and beyond its boundary. In application to PSR B0656+14 the maximum critical frequency is $4 \times 10^{15} \mathrm{~Hz}$ and the optical luminosity $\sim 10^{28} \mathrm{ergs} \mathrm{s}^{-1}$. In case of Crab pulsar, the optical emission comes from the lower part of the resonance region, where $\psi<\theta$ and $p \sim p_{0}$. The optical luminosity can be as large as $\sim 10^{34}$ ergs $^{-1}$. The spectrum of emission from the upper part of the resonance region extends up to $10 \mathrm{MeV}$.

\section{Conclusions}

The model suggested can reproduce the general features of non-thermal emission of both young and middle-aged pulsars and implies a physical connection between the radio and high-energy emission of pulsars. As is known from observations (Goldoni et al. 1995), higher efficiencies of conversion of pulsar rotational energy into high-energy radiation are indeed accompanied by lower radio efficiencies. Furthermore, the recent simultaneous radio and optical observations of giant pulses from the Crab pulsar (Shearer et al. 2003) also testify to the indirect link of radio and higher-energy emission. A search for other observational manifestations of such a link would be of considerable interest.

\section{References}

Goldoni, P., Musso, C., Caraveo, P. A., \& Bignami, G. F. 1995, A\&A, 298, 535 Lyubarskii, Y. E., \& Petrova, S. A. 1998, A\&A, 337, 433

Petrova, S. A. 2002, MNRAS, 336, 774

Petrova, S. A. 2003, MNRAS, 340, 1229

Petrova, S. A. 2004, in preparation

Shearer, A. et al. 2003, Science, 301, 493 